\title{
Associação entre consumo alimentar e (in)segurança alimentar e nutricional em São José dos Ramos - PB
}

\author{
Association between consumption and food (in)security \\ in food and nutrition of São José dos Ramos - PB
}

\section{Autores | Authors}

\Cleyton Cézar Souto SILVA

Universidade Federal da Paraíba (UFPB) Centro de Ciências Exatas e da Natureza

Departamento de Estatística

Mestrado em Modelos de Decisão e Saúde

Rua Coronel Souza Lemos, 307, Miramar CEP: 58043-190

João Pessoa/PB - Brasil

e-mail:cez2004@hotmail.com

\section{Karen Beatriz Borges OLIVEIRA Aline da Silva ALVES José Anael NEVES \\ Cely Alana Carvalho MODESTO Rodrigo Pinheiro de Toledo \\ VIANNA \\ Universidade Federal da Paraíba (UFPB) \\ Centro de Ciências da Saúde Departamento de Nutrição João Pessoa/PB - Brasil e-mail:karenbeatriz_04@hotmail.com alinealves280@yahoo.com.br anaelneves@gmail.com celymodesto@hotmail.com vianna-rodrigo@yahoo.com.br}

Autor Correspondente / Corresponding Author Publicado / Published: dezembro/2012

\section{Resumo}

A prevalência de insegurança alimentar é importante para a avaliação das condições de vida e para o planejamento de políticas públicas de combate à fome. A pesquisa foi realizada com 177 famílias de São José dos Ramos-PB, no ano de 2005, por meio de entrevista. A análise dos dados, por regressão logística e ODDS RATIO, utilizou como variável dependente estar ou não em segurança alimentar, avaliação resultante da aplicação da EBIA; e, como variável independente dicotômica, o consumo de 15 conjuntos de alimentos. O modelo em regressão logística e o odds ratio mostraram associação de leite integral, manteiga, biscoito, bolacha e bolo com a segurança alimentar. Contudo, deve-se levar em consideração o conceito ampliado de Segurança Alimentar e Nutricional, que enfatiza outros fatores relevantes além do consumo alimentar, como a disponibilidade de alimento, que se incorpora às condições de renda e padrão de vida.

Palavras-chave: Segurança alimentar; Consumo alimentar; Direito humano à alimentação.

\section{Summary}

The prevalence of food insecurity is important for the assessment of the conditions of life and planning of public policies for combating hunger. The survey was conducted with 177 families of São José dos Ramos, in the year 2005, through interview. Data analysis, through logistics regression and ODDS RATIO, used as the dependent variable is in food safety assessment resulting from the application of EBIA, and as a variable dichotomic-independent consumption of 15 sets of food. The regression model Logistics and the odds ration of Association showed whole milk; butter, biscuit, cracker, cake with food safety. However you should take into consideration the expanded concept food and nutritional security that emphasizes other relevant factors beyond the food consumption as food availability, which incorporates income conditions and standard of living.

Key words: Food safety; Food consumption; Human right to food. 


\section{Introdução}

Segundo a II Conferência Nacional de Segurança Alimentar e Nutricional (CONSEA), o conceito de Segurança Alimentar e Nutricional (SAN) foi incorporado na Lei Orgânica de Segurança Alimentar e Nutricional (LOSAN) (Lei n. ${ }^{\circ} 11.346$, de 15 de julho de 2006), tendo sido objeto de discussão aprofundada nas III e IV Conferências. Esse conceito é definido como a realização do direito de todos ao acesso regular e permanente a alimentos de qualidade, em quantidade suficiente, sem comprometer o acesso a outras necessidades essenciais, tendo como base práticas alimentares promotoras da saúde, que respeitem a diversidade cultural e que sejam ambiental, econômica e socialmente sustentáveis (CONSEA, 2004).

A insegurança alimentar (IA) existe quando a disponibilidade de alimentos nutricionalmente adequados e seguros, ou a capacidade para adquirir esses alimentos de forma socialmente aceitável, é limitada ou incerta. A insegurança alimentar pode variar do medo de não ser capaz de obter o alimento à fome em razão da escassez de alimentos (EL-SAYED et al., 2010; MILLER et al., 2011).

Também a vulnerabilidade social está diretamente relacionada à $I A$, podendo esta resultar de uma combinação de fatores que podem produzir uma deterioração do nível de bem-estar de pessoas, famílias ou comunidades, conforme a exposição a determinados fatores sociais. Uma causa fundamental para este tipo de problema é a incapacidade de acesso, especialmente pela falta de poder aquisitivo para adquirir os alimentos de que se necessita (WILLOWS et al., 2011).

É a baixa renda ou a pobreza que limitam a aquisição de alimentos. Nestas circunstâncias, vários estudos têm demonstrado a concordância da IA com a instabilidade no emprego, a insuficiência de renda e o baixo nível de escolaridade dos pais de família, implicando graus variáveis de comprometimento na aquisição e no consumo de um ou de vários alimentos em relação a um ou vários membros da família (OLIVEIRA et al., 2010; RANSEY et al., 2012).

Assim, o direito humano a uma alimentação adequada disponível, parte integrante dos direitos fundamentais da humanidade, é definido pelo pacto mundial do qual o Brasil é signatário, sendo que, por meio da lei Orgânica de Segurança Alimentar e Nutricional, é proposta a busca pela qualidade, pelo acesso e pela variedade de alimentos à grande maioria da população. Para a realização deste objetivo, foram criados os programas governamentais a exemplo do Programa Bolsa Família, que proporciona certa quantia em dinheiro às famílias para que estas possam ter uma renda a mais para suas despesas (GUBERT et al., 2010).
Por isso, conhecer a prevalência de IA é de grande importância para a avaliação das condições de vida e, consequentemente, para o planejamento de políticas públicas de combate à fome. A Pesquisa Nacional por Amostras Domiciliares (PNAD), conduzida pelo Instituto Brasileiro de Geografia e Estatística (IBGE) no ano de 2004 (IBGE, 2004a), utilizando a Escala Brasileira de Insegurança Alimentar (EBIA), identificou que $34,8 \%$ da população brasileira sofre com algum grau de IA, representando um importante problema de saúde pública. A prevalência de IA grave, entretanto, apresentou variações de acordo com as regiões do País, com destaque para aquelas em piores condições: Norte (12,4\%) e Nordeste (10,9\%), atingindo 15,0\% das famílias no Estado da Paraíba (IBGE, 2004b).

Considerado um Estado de médio desenvolvimento pelo Programa das Nações Unidas (PNUD), a Paraíba encontra-se em uma situação não favorável, demonstrada pela pesquisa realizada por Viana e Segall-Corrêa (2008), a qual destacou, dentre os municípios pesquisados, São José dos Ramos, que é apresentado como o de menor índice de SA $(30,2 \%)$ e o de maior índice de IA grave $(22,8 \%)$. Este estudo seccional aplicou a EBIA como instrumento de mensuração da situação familiar em 14 municípios do Estado, apresentando como resultado um nível de insegurança grave, que variou entre 5,4\% e $22,8 \%$.

Essa investigação desagregada em municípios do interior do Estado permitiu conhecer características locais que podem não ser percebidas em unidades de análise regionais ou estaduais. Estas características, comuns à grande maioria dos municípios do semiárido nordestino, mostram as consequências das desigualdades regionais e possibilitam dimensionar o contingente populacional sob risco de ocorrência de problemas nutricionais (VIANA e SEGALL-CORRÊA, 2008).

Dados municipais de prevalência de IA são, portanto, necessários à execução da gestão pública, pois existem importantes discrepâncias sociais e econômicas intraestaduais que se manifestam nos municípios. Sabendo-se que este problema é parcialmente oriundo desses determinantes, esperam-se diferenças importantes na sua ocorrência em nível municipal.

Burlandy e Costa (2007) destacaram que o conceito ampliado de SAN articula a dimensão alimentar, que engloba a produção, a comercialização e o consumo de alimentos, e a dimensão nutricional, referente à utilização do alimento pelo organismo e sua relação com a saúde. Assim, a avaliação do consumo alimentar representa um papel importante na área de pesquisa em nutrição e saúde, podendo influenciar, consequentemente, no desenvolvimento de programas referentes a esse campo (CAVALCANTE et al., 2004). 
O tipo de consumo alimentar de uma determinada população é influenciado por fatores econômicos, sociais, nutricionais e culturais. Desta forma, é importante verificar também a qualidade da alimentação dos cidadãos, que devem consumir alimentos seguros, que satisfaçam suas necessidades nutricionais, respeitando seus hábitos alimentares e culturais, e que promovam sua saúde (MALUF et al., 1996; OLIVEIRA e THÉBAUD-MONY, 1998).

Uma alimentação inadequada, quantitativa ou qualitativamente, revela um comprometimento do aporte de energia e nutrientes, intensificando a deficiência deste aporte e refletindo no déficit de crescimento de crianças. Dessa forma, considerando-se que a influência da insegurança alimentar no consumo de alimentos representa um importante pilar na investigação da situação nutricional, notou-se a importância de se avaliar a associação entre a segurança alimentar e algumas variáveis sobre o consumo alimentar das famílias moradoras de São José dos Ramos. Assim, por meio de um modelo explicativo de regressão logística, com base nos dados coletados na pesquisa de Viana e SegallCôrrea (2008), buscou-se avaliar a associação entre segurança alimentar e determinadas variáveis.

\section{Material e métodos}

Este estudo é caracterizado como transversal, exploratório, descritivo e de abordagem quantitativa. Foram utilizados os dados de 2005, obtidos a partir do banco de dados da pesquisa "Insegurança alimentar das famílias residentes em municípios do interior do Estado da Paraíba". Essa pesquisa foi realizada em 14 municípios do Estado da Paraíba, onde foi iniciado o programa Fome Zero do Governo Federal, tendo sido identificados como os mais carentes do semiárido paraibano. Eles estão localizados em quatro regiões do Estado: o Agreste Paraibano, a Mata Paraibana, a Borborema e o Sertão (VIANA e SEGALL-CÔRREA, 2008).

O local escolhido para este estudo foi o município de São José dos Ramos, na região da Mata Paraibana, com população de 5.508 habitantes, tendo 57\% das famílias vivendo na zona rural; este município foi escolhido por apresentar o menor índice de SA, segundo os resultados da pesquisa realizada por Viana e SegallCorrêa (2008).

A unidade amostral é composta pelas famílias moradoras tanto da zona rural como da zona urbana deste município e que recebiam o auxílio do Programa Bolsa Família.

A amostra foi definida por amostragem aleatória estratificada, em que o município foi dividido por área urbana e área rural, tendo sido os tamanhos das amostras nos estratos calculados adotando-se partilha proporcional, com base nos dados populacionais do censo demográfico de 2000. Utilizou-se erro amostral máximo de 5\% sob nível de confiança de 95\%. Esta estimativa gerou os tamanhos de amostra para São José dos Ramos de 287 domicílios (105 urbanos e 182 rurais).

A coleta de dados, realizada em 2005, utilizou entrevistas domiciliares por entrevistadores previamente treinados, em que cada responsável da família foi convidado a participar da pesquisa; em caso de aceitação, este assinou uma carta de Consentimento Livre e Esclarecido.

O instrumento de coleta de dados foi composto pelos seguintes módulos:

- Caracterização da família: identificação do chefe da família, total de moradores por domicílio segundo idade, gênero, escolaridade e ocupação de cada morador;

- Dados sociodemográficos: local de residência, tipo de construção de moradia e acesso a serviços de água, esgoto e coleta de lixo;

- Escala Brasileira de Insegurança Alimentar (EBIA): 15 perguntas, destinadas às famílias com algum morador menor de 18 anos, ou nove perguntas para famílias compostas somente por adultos, com graus de gravidade crescente, indo desde a preocupação com a falta de alimento no domicílio até a situação de algum morador haver passado um dia inteiro sem comer nos três meses anteriores à pesquisa;

- Afirmativas sobre problemas relacionados à insegurança alimentar: idosos, hipertensão e diabetes, quantidade e variedade de alimentos;

- Informações de rendimentos familiares: renda de cada morador e a participação em programas de doação de alimentos ou complementação de renda; neste caso, com a quantificação do valor monetário recebido; e

- Consumo alimentar: diversos tipos de alimentos divididos em 15 grupos, informando frequência, período e porção do consumo de 1) Leite integral (puro/com café), Leite desnatado (puro/com café); 2) Queijo prato, mussarela e manteiga; 3) Pão francês, Pão de fôrma, Pão doce; 4) Biscoito, bolacha salgada ou doce, torradas; 5) Farofa, farinha de milho, farinha de mandioca; 6) Embutidos (linguiça, salsicha); 7) Presunto, apresuntado, mortadela e outros frios; 8) Ovos; 9) Açúcar em sucos, café, café com leite; 10) Doce de abóbora, batata-doce, caju, goiabada, marmelada; 11) Margarina comum passada no pão, biscoito, bolacha, bolo; 12) Manteiga passada no pão, biscoito, bolacha, bolo; 13) Aveia ou farinha láctea; 14) frutas, sem incluir o suco; 15) Vegetais (verduras, legumes), sem incluir batatas. 
Para montagem do banco de dados para análise, neste novo estudo, foram utilizados dados de 177 famílias que responderam ao modulo de consumo alimentar do instrumento de coleta de dados. $\mathrm{Na}$ análise dos dados, foi utilizado como variáveis independentes dicotômicas o consumo ou não dos 15 conjuntos de alimentos, e, como variável dependente, estar ou não em segurança alimentar, decisão esta resultante da aplicação da EBIA.

Conforme esta escala, quando o entrevistado responde negativamente a todas as questões, a família é classificada em situação de segurança alimentar; até cinco respostas positivas, insegurança alimentar leve, o que representa restrição na qualidade dos alimentos consumidos; de seis a dez respostas positivas, insegurança alimentar moderada, representando restrição na quantidade de alimentos, e de 11 a 15 respostas positivas, insegurança alimentar grave, que representa um estado no qual a família convive com a situação real de fome, na qual os adultos e/ou as crianças residentes nesses domicílios deixam de realizar refeições ou mesmo ficam até um dia inteiro sem comida. Para famílias somente com adultos, a escala classifica em segurança alimentar quando todas as respostas são respondidas negativamente; em insegurança alimentar leve: de uma a três respostas positivas; insegurança alimentar moderada: de quatro a seis respostas positivas; insegurança alimentar grave: de sete a nove respostas positivas (VIANA e SEGALL-CÔRREA, 2008).

Para análise estatística dos dados, foi utilizada a técnica da regressão logística, principalmente pela facilidade de interpretação dos parâmetros de um modelo logístico e também pela possibilidade do uso desse tipo de metodologia mesmo quando a resposta de interesse não é originalmente do tipo binário, podendo-se dicotomizar a resposta de modo que a probabilidade de sucesso possa ser ajustada (PAULA, 2010).

Para obtenção de um modelo explicativo sem variáveis "dummies", foi utilizado o método backward no nível de significância de $5 \%$, ou seja, as variáveis independentes de consumo alimentar iam sendo eliminadas do modelo de acordo com o nível de confiança de $95 \%$ ( $p>0,05)$, até a composição do modelo final, indicando os alimentos que apresentam associação significativa com a segurança alimentar.

Foi realizado o teste de normalidade do modelo final por meio do gráfico de quantil-quantil (também conhecido com Q-Q plot) e com o auxílio de envelopes simulados, mostrando a fidedignidade do mesmo. A razão de chances (ODDS RATIO), juntamente com seu intervalo de confiança no nível de 95\%, foi utilizada para medir a chance de uma família com SA consumir o alimento que obteve associação significativa no modelo de regressão logística.
Toda a análise estatística foi realizada no Software estatístico livre R, de acesso gratuito no site www.r-projet. org. Foram seguidos os preceitos éticos de acordo com a resolução 196/96 do Conselho Nacional de Saúde (MS), com aprovação do Comitê de Ética em Pesquisa do Centro de Ciências da Saúde da Universidade Federal da Paraíba.

\section{Resultados e discussão}

Após classificação feita através da EBIA, constatou-se que, das famílias incluídas no banco de dados desta análise, 135 apresentavam insegurança alimentar em algum dos níveis (leve, moderado ou grave) e 42 encontravam-se em situação de segurança alimentar.

A Tabela 1 apresenta os grupos de alimentos que não apresentaram associação significativa no nível de confiança de $95 \%$, com seus respectivos p-valores:

Corroboram para estes resultados as análises de Levy et al. (2010), ao encontrarem menor consumo de hortaliças e frutas entre seus estudantes pesquisados. Embora Antunes et al. (2010) tenham demonstrado em seus achados que crianças com insegurança alimentar leve apresentaram consumo significativamente maior das porções dos grupos das hortaliças, quando comparadas às crianças de famílias em situação de segurança alimentar. Entre as crianças de famílias com as formas moderada e grave de IA, o consumo médio de porções apresentou-se significativamente reduzido para cereais, hortaliças, frutas, carnes, ovos e gorduras.

Tabela 1. Coeficientes estimados do modelo de regressão logística para consumo alimentar e segurança alimentar sem associação estatisticamente significativa.

\begin{tabular}{lccrl} 
Variáveis & $\begin{array}{c}\text { Valor } \\
\text { estimado }\end{array}$ & $\begin{array}{c}\text { Crientes } \\
\text { padrão }\end{array}$ & valor Z & P-valor \\
\hline Fruta & 0.02739 & 0.48083 & 0.057 & 0.95458 \\
Embutidos & -0.1010 & 0.4441 & -0.227 & 0.82012 \\
Leite & 0.1965 & 0.5309 & 0.370 & 0,711321 \\
desnatado & & & & \\
Vegetais & 0.1754 & 0.4444 & 0.395 & 0.69307 \\
Presunto & -0.2584 & 0.4250 & -0.608 & 0.543217 \\
Margarina & 0.3372 & 0.4954 & 0.681 & 0.496034 \\
Farinha & -0.2988 & 0.4452 & -0.671 & 0.502169 \\
Doce & -0.3259 & 0.4177 & -0.780 & 0.435300 \\
Aveia & -0.6197 & 0.6983 & -0.888 & 0.37480 \\
Ovo & -0.5327 & 0.5214 & -1.022 & 0.306906 \\
Queijo & 0.5211 & 0.4124 & 1.264 & 0.206381 \\
Biscoito & 0.9310 & 0.6647 & 1.401 & 0.16130 \\
Açúcar & -0.4781 & 0.4774 & -1.001 & 0.31668 \\
Pão doce & 0.7432 & 0.4299 & 1.729 & 0.083862 \\
\hline Parametroded
\end{tabular}

Parâmetro de dispersão para a família binominal tomada para 1; desvio nulo: 243,99 em 176 graus de liberdade; desvio residual: 178,87 em 173 graus de liberdade; número de interações Fisher de pontuação: 4. 
Panigassi et al. (2008), em análise do consumo de alimentos reguladores (frutas, verduras e legumes), ricos em fibras, vitaminas e minerais, entre os membros de famílias em IA leve, obtiveram em seus resultados um consumo baixo: 35\% dos entrevistados não incluíam diariamente legumes e verduras em suas dietas e $56 \%$ não consumiam frutas. Ao compararem o consumo alimentar entre os três níveis de $\mathrm{IA}$, encontraram uma forte e significante tendência decrescente de consumo de carne, leite, derivados do leite, frutas e verduras/legumes, como também de doces e refrigerantes, na mesma tendência. Houve, por outro lado, maior consumo de todos esses grupos entre os informantes das famílias em SA, sendo menor entre aquelas em IA leve e diminuindo mais entre as que apresentaram IA moderado e grave.

Em pesquisa realizada com famílias que possuem na sua composição membros de 65 anos e mais, observou-se maior consumo, em ordem crescente, de verduras, leite, frutas, carne, derivados de leite e doces nos grupos familiares em SA do que em algum nível de IA, havendo ainda uma maior chance de consumo desses alimentos entre os entrevistados de famílias em segurança alimentar (OR e IC95\% maior que 1) (MARÍN-LEÓN et al., 2005).

Esses mesmos autores ainda constataram que a deficiência qualitativa de alimentos do membro entrevistado aumenta na medida em que aumenta o nível de insegurança alimentar. Assim, mesmo em uma parcela das famílias com segurança, não se observou consumo diário de carnes, frutas e verduras, sendo o ovo pouco consumido, independentemente do nível de insegurança alimentar.

De acordo com a análise estatística da Tabela 2, os alimentos que apresentaram associação significativa

Tabela 2. Coeficientes estimados do modelo de regressão logística para consumo alimentar e segurança alimentar com associação estatisticamente significativa.

\begin{tabular}{lcccc}
\multicolumn{5}{c}{ Coeficientes } \\
\multicolumn{1}{c}{ Variáveis } & $\begin{array}{c}\text { Valor } \\
\text { estimado }\end{array}$ & $\begin{array}{c}\text { Erro } \\
\text { padrão }\end{array}$ & valor Z & P-valor \\
\hline $\begin{array}{l}\text { Leite integral } \\
\text { (puro ou com } \\
\text { café) }\end{array}$ & 0.7682 & 0.3858 & 2.009 & $0.04506^{*}$ \\
$\begin{array}{l}\text { Pão francês, } \\
\text { pão de forma }\end{array}$ & -1.1440 & 0.2936 & -3.897 & $9.76 \mathrm{e}-05^{\star \star *}$ \\
$\begin{array}{l}\text { Manteiga } \\
\text { passada no } \\
\text { pão, biscoito, } \\
\text { bolacha, bolo }\end{array}$ & -1.1006 & 0.3858 & -2.853 & $0.00433^{\star *}$ \\
\hline
\end{tabular}

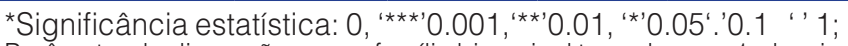
Parâmetro de dispersão para a família binominal tomada para 1; desvió nulo: 243,99 em 176 graus de liberdade; desvio residual: 178,87 em 173 graus de liberdade; número de interações Fisher de pontuação: 4. com a segurança alimentar foram: Leite integral (puro/com café); Pão francês, Pão de fôrma; Manteiga passada no pão, biscoito, bolacha, bolo. Isso quer dizer que a família que está em segurança alimentar tem a presença destes alimentos no consumo alimentar de sua casa.

Panigassi et al. (2008) corroboram com este resultado ao demonstrar em sua pesquisa que existe um maior consumo, entre os grupos familiares que estão em $\mathrm{SA}$, de alimentos construtores ricos em proteínas, como o leite e seus derivados. Diversamente, Fávaro et al. (2007) encontraram um alto consumo de leite em famílias indígenas com IA moderado/grave, que pode ser justificado em função da distribuição de leite em pó, realizada pela Fundação Nacional de Saúde (FUNASA). $\mathrm{Na}$ Paraíba (PB), 52\% da sua população pesquisada por Teixeira e Silva (2007) consumia diariamente este alimento.

O leite e seus derivados são considerados alimentos marcadores de alimentação saudável. Esta classificação foi baseada nas recomendações nutricionais para prevenção de doenças crônicas não transmissíveis e, também, em evidências que sugerem a associação de uma alimentação não saudável com o excesso de peso e outros fatores de risco para doenças crônicas não transmissíveis, como as dislipidemias (WHO, 2004).

Em um estudo realizado com alunos do $9^{\circ}$ ano do Ensino Fundamental de escolas públicas e privadas das 26 capitais de estados brasileiros e do Distrito Federal, no tocante ao consumo regular de leite, observou- se maior proporção entre alunos de escola privada do que entre alunos de escola pública, além da associação direta dessa prática com a escolaridade materna (LEVY et al., 2010).

Em contraposição, Antunes et al. (2010), em uma análise por meio dos grupos de alimentos, revelaram em sua pesquisa que as crianças não atingem as recomendações do consumo das porções de alimentos com proteínas de alto valor biológico (leites e derivados) e hortaliças, de acordo com as recomendações, e elas apresentam, ainda, consumo elevado dos alimentos de alta densidade calórica (gorduras e açúcares).

Por sua vez, Marín-Léon et al. (2005) demonstraram em sua pesquisa haver diferença de consumo de derivados do leite em famílias em segurança alimentar, ao apontarem que 51,8\% não consomem tais derivados, entre famílias com entrevistado idoso, contra 29,7\%, quando o entrevistado não é idoso.

Além de ser demonstrado quão importante para a segurança alimentar é o consumo de leite e seus derivados, como a manteiga, este é considerado um alimento completo e de elevado valor biológico na alimentação, oferecendo ao indivíduo macro e micronutrientes essenciais ao organismo. Assim, deve ser 
um componente fundamental da dieta, especialmente na alimentação de crianças e idosos, por ser uma excelente fonte de vitamina $A$ e das vitaminas do complexo $B$, como a tiamina (B1), a riboflavina (B2) e a cobalamina (B12). $A$ vitamina $D$ e o ácido fólico aparecem em menores quantidades (SANTOS, 2008).

Em termos de minerais, os mais abundantes no leite e derivados são: o cálcio, que contribui para o desenvolvimento dos dentes, para a formação óssea e para a prevenção da osteoporose; o potássio, importante no equilíbrio ácido-básico do organismo e no transporte de oxigênio; o fósforo, relacionado à constituição dos dentes, além de atuar na formação dos ácidos nucleicos, e o sódio, relacionado com o controle da pressão arterial (SANTOS, 2008).

De acordo com Franco (2007), os pães, biscoitos e bolos fazem parte do grupo de cereais que representam a principal fonte de energia para o organismo, compostos por hidratos de carbono ou glicídios, sendo que seu valor calórico total na dieta, em algumas regiões do mundo, constitui-se quase como fonte exclusiva. Além dessa característica, estes alimentos exercem a função de poupança sobre as proteínas, transformando-se em gordura, quando em excesso na alimentação.

Segundo estudo realizado por Antunes et al. (2010), crianças com insegurança alimentar leve apresentaram consumo significativamente maior das porções dos grupos dos doces e açúcares, e consumo menor para os cereais, quando comparadas às crianças de famílias em situação de segurança alimentar. Entre as crianças de famílias com as formas moderada e grave de insegurança alimentar, o consumo médio de porções apresentou-se significativamente reduzido para cereais e gorduras.

Levy et al. (2010), em sua pesquisa com estudantes, observaram que, entre as meninas, houve uma maior proporção de consumo regular de guloseimas, biscoitos doces e embutidos, com associação direta deste consumo com a escolaridade materna.

No Brasil, observou-se a transformação nos hábitos alimentares da população com o aumento do consumo de óleos vegetais e margarina, açúcar e, em geral, alimentos com alta densidade energética, como biscoitos e refrigerantes, conforme observado na Pesquisa de Orçamentos Familiares (POF) de 2003.

Em relação ao grupo dos cereais, Teixeira e Silva (2007) relataram consumo diário por $64 \%$ dos entrevistados no Estado da Paraíba; Karam e Barboza (2010) obtiveram $35,4 \%$ dos entrevistados consumindo dois pães por dia, $29,2 \%$ consumindo um pão por dia e 20,8\%, três pães por dia. Uma dieta pobre em carboidratos complexos e rica em açúcares simples e gorduras está associada à obesidade e a outras doenças crônicas, como diabetes mellitus tipo 2 e hipertensão arterial, levando à perda da qualidade de vida e, consequentemente, a um maior risco de morbimortalidade, principalmente por doenças cardiovasculares (PANIGASSI et al., 2008).

O modelo final explicativo em regressão logística ajustado para consumo alimentar foi $Y=0,76 . X 1-1,14$. X2 - 1,10.X3. O Null deviance (176 graus de liberdade) próximo do Residual deviance (173) indica que o modelo é adequado.

Por meio do gráfico de quantil-quantil (também conhecido com Q-Q plot) e com o auxílio de envelopes simulados, pode-se concluir que o modelo satisfaz as suposições de normalidade exigidas e, portanto, a inferência sobre o mesmo está correta (Figura 1).

A Tabela 3 mostra a razão de chances brutas (ODDS RATIO) para o consumo de alimentos do modelo que tiveram associação significativa com seus respectivos intervalos de confiança:

Pela análise do ODDS RATIO, as famílias que estão em segurança alimentar têm duas vezes mais a chance de ter em seu consumo alimentar o leite integral puro ou com café das que não estão em SA. E possuem três vezes mais a chance de ter no consumo o pão francês e/ou pão de fôrma, como também de manteiga passada no pão, biscoito, bolacha e bolo do que aquelas famílias em situação contrária.

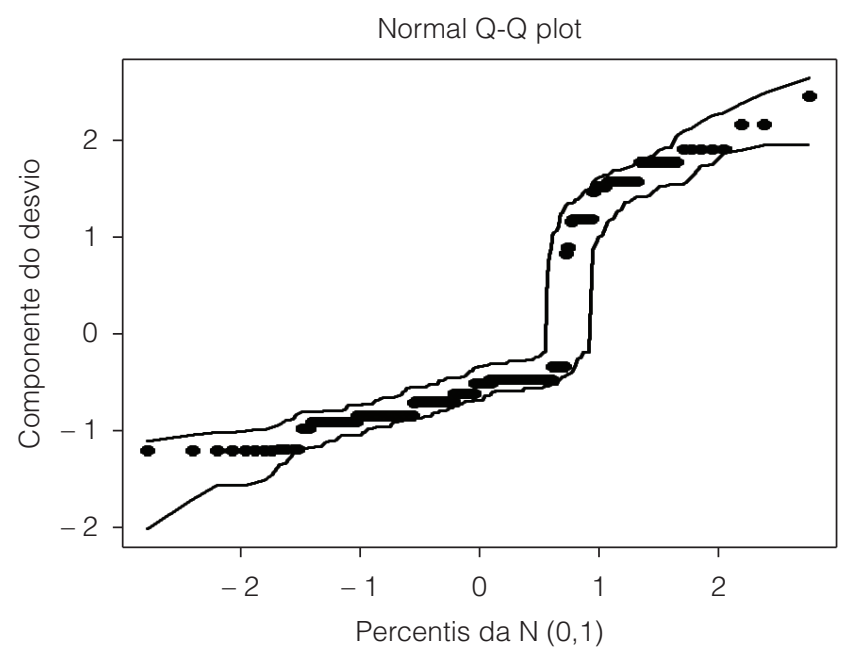

Figura 1. Gráfico da normalidade (quantil-quantil).

Tabela 3. Razão de chances brutas e intervalos de confiança para o consumo de alimentos do modelo final.

\section{Variáveis}

OR Bruta IC $\mathbf{9 5 \%}$

Leite integral (puro ou com café)

2.15

$1.01-4.59$

Pão francês, pão de fôrma

0.31

$0.17-0.56$

Manteiga passada no pão,

biscoito, bolacha, bolo
0.33 
Associação entre consumo alimentar e (in)segurança alimentar e nutricional em São José dos Ramos - PB SILVA, C. C. S. et al.

\section{Conclusão}

A pesquisa mostrou que alguns alimentos são essenciais para prevenir a IA; porém, levando-se em consideração o conceito ampliado de (In)Segurança Alimentar e Nutricional, é importante enfatizar que outros fatores, além do consumo alimentar, se tornam relevantes, como a disponibilidade de alimento, que se incorpora às condições de renda e padrão de vida. Outro fator relevante no conceito ampliado de SA se refere ao respeito à diversidade cultural.

Este estudo poderá fornecer subsídios para o desenvolvimento e o aperfeiçoamento de políticas sociais e econômicas para a melhoria das condições e perspectivas de vida da população, principalmente dos estratos em maior risco de insegurança alimentar. Esta melhoria é essencial para a garantia do direito humano à alimentação adequada, bem como dos demais direitos humanos, e só será efetuada com o compromisso ético e ativo dos gestores públicos e com a participação e o controle social efetivo e qualificado.

Dessa forma, o modelo explicativo proposto mostrou-se adequado e poderá ainda ser melhorado com a utilização de um banco de dados sobre consumo alimentar com informações atualizadas, em novas coletas de dados sobre SA no município de São José dos Ramos-PB.

\section{Referências}

ANTUNES, M. M. L.; SICHIERI, R.; SALLES-COSTA, R. Consumo alimentar de crianças menores de três anos residentes em área de alta prevalência de insegurança alimentar domiciliar. Cadernos de Saúde Pública, Rio de Janeiro, v. 26, n. 8, p. 16421650, 2010.

BURLANDY, L.; COSTA, R. S. Segurança alimentar e nutricional: concepções e desenhos de investigação. In: KAC, G.; SICHIERI, R.; GIGANTE, D. P. Epidemiologia Nutricional. Rio de Janeiro: Ed. Fiocruz, 2007. p. 485-502.

CAVAlCANTE, A. A. M.; PRIORE, S. E.; FRANCESCHINI, S. C. C. Estudos de Consumo Alimentar: Aspectos Metodológicos Gerais e o seu Emprego na Avaliação de Crianças e Adolescentes. Revista Brasileira de Saúde Materno-Infantil, Recife, v. 4, n. 3, p. 229-240, 2004. http://dx.doi.org/10.1590/S151938292004000300002

CONSELHO NACIONAL DE SEGURANÇA ALIMENTAR E NUTRICIONAL - CONSEA. Documento base. In: CONFERÊNCIA NACIONAL DE SEGURANÇA ALIMENTAR E NUTRICIONAL, 2., 2004, Brasília. Anais... Brasília: Consea, 2004.

EL-SAYED, A. M.; HADLEY, C.; TESSEMA, F.; TEGEGN, A.; COWAN, J. A.; GALEA, S. Household food insecurity and symptoms of neurologic disorder in Ethiopia: An observational analysis. BMC Public Health, London, v. 10, p. 802, 2010. PMid:21194486. PMCid:3027184. http://dx.doi. org/10.1186/1471-2458-10-802

INSTITUTO BRASILEIRO DE GEOGRAFIA E ESTATÍSTICA - IBGE. Pesquisa de orçamentos familiares: 2002-2003. Resultados preliminares. Rio de Janeiro: IBGE; 2004a.

INSTITUTO BRASILEIRO DE GEOGRAFIA E ESTATÍSTICA - IBGE. Pesquisa de Orçamentos Familiares 2002-2003. Resultados da Amostra do Censo Demográfico 2000. Rio de Janeiro: IBGE; 2004b. Disponível em: <www.ibge.gov.br>. Acesso em: 26 mar. 2012

FRANCO, G. Tabela de Composição Química dos Alimentos. São Paulo: Atheneu, 2007. 307 p.

FÁVARO, T.; RIBAS, D. L. B.; ZORZATTO, J. R.; SEGALLCORRÊA, A. M.; PANIGASSI, G. Segurança alimentar em famílias indígenas Térena, Mato Grosso do Sul, Brasil. Cadernos de Saúde Pública, Rio de Janeiro, v. 23, n. 4, p. 785-793, 2007. http://dx.doi.org/10.1590/S0102-311X2007000400006

GUBERT, M. B.; BENÍCIO, M. H. A.; SANTOS, L. M. P. Estimativas da insegurança alimentar Grave nos municípios brasileiros. Cadernos de Saúde Pública, Rio de Janeiro, v. 26, n. 8, p. 1595-1605, 2010.

KARAM, K. M.; BARBOZA, L. M. V. Estudos dos Hábitos Alimentares na Educação de Jovens e Adultos. Portal da Secretaria da Educação do Paraná, 2010. Disponível em: <www. diaadiaeducacao.pr.gov.br/portals/pde/arquivos/968-4.pdf>. Acesso em: 26 de mar. 2012.

LEVY, R. B.; CASTRO, I. R. R.; CARDOSO, L. O.; TAVARES, L. F.; SARDINHA, L. M. V.; GOMES, F. S.; COSTA, A. W. N. Consumo e comportamento alimentar entre adolescentes brasileiros: Pesquisa Nacional de Saúde do Escolar (PeNSE), 2009. Ciência e Saúde Coletiva, Rio de Janeiro, v. 15, p. 3085-3097, 2010. Suplemento 2. http://dx.doi.org/10.1590/ S1413-81232010000800013

MALUF, R. S.; MENEZES, F.; VALENTE, F. Contribuição ao tema da segurança alimentar no Brasil. Revista Cadernos de Debate, Campinas, v. 4, p. 66-68, 1996.

MARÍN-LEÓN, L.; SEGAL-CORRÊA, A. M.; PANIGASSI, G.; MARANHA, L. K.; SAMPAIO, M. F. A.; PÉREZ-ESCAMILLA, R. A percepção de insegurança alimentar em famílias com idosos em Campinas, São Paulo, Brasil. Cadernos de Saúde Pública, Rio de Janeiro, v. 21, n. 5, p. 1433-1440, 2005. http://dx.doi. org/10.1590/S0102-311X2005000500016

MILLER, C. L.; BANGSBERG, D. R.; TULLER, D. M.; SENKUNGU, J.;KAWUMA, A.; FRONGILLO, E. A.; WEISER, S. D. Food Insecurity and Sexual Risk in an HIV Endemic Community in Uganda. Aids and Behavior, New York, v. 15, n. 7 , p. 1512-1519, 2011. PMid:20405316. PMCid:3110536. http:// dx.doi.org/10.1007/s10461-010-9693-0

OLIVEIRA, J. S.; LIRA, P. I. C.; OSÓRIO, M. M.; SEQUEIRA, L. A. S.; COSTA, E. C.; GONÇALVES, F. C. L. S. P.; BATISTA FILHO, M. 
Associação entre consumo alimentar e (in)segurança alimentar e nutricional em São José dos Ramos - PB SILVA, C. C. S. et al.

Anemia, hipovitaminose A e insegurança alimentar em crianças de municípios de Baixo Índice de Desenvolvimento Humano do Nordeste do Brasil. Revista Brasileira de Epidemiologia, São Paulo, v. 13, n. 4, p. 651-664, 2010. PMid:21180854. http:// dx.doi.org/10.1590/S1415-790X2010000400010

OLIVEIRA, S. P.; THEBAUD-MONY, A. Estudo do Consumo Alimentar: em busca de uma abordagem multidisciplinar. Revista de Saúde Publica, São Paulo, v. 31, n. 2, p. 202-207, 1998. http:// dx.doi.org/10.1590/S0034-89101997000200015

PAULA, G. A. Modelos de Regressão com Apoio Computacional. São Paulo: Instituto de Matemática e Estatística, Universidade de São Paulo, 2010.

PANIGASSI, G.; SEGALL-CORRÊA, A. M.; MARIN-LEÓN, L.; PÉREZ-ESCAMILLA, R.; MARANHA, L. K.; SAMPAIO, M. F. A. Insegurança alimentar intrafamiliar e perfil de consumo de alimentos. Revista de Nutrição, Campinas, v. 21, p. 135-144, jul./ago. 2008. Suplemento. http://dx.doi.org/10.1590/S141552732008000700012

RANSEY, R.; GISKES, K.; TURRELL, G.; GALLEGOS, D. Food insecurity among adults residing in disadvantaged urban areas: potential health and dietary consequences. Public Health Nutrition, Wallingford, v. 15, n. 2, p. 227-37, 2012. http://dx.doi. org/10.1017/S1368980011001996
SANTOS, L. C. Análise da Qualidade Microbiológica e Físico-Química do Leite Pasteurizado Tipo C Padronizado, Envasado e Comercializado na Cidade de Pará de Minas-MG e sua Influência na Saúde do Consumidor. Pará de Minas: Conut, 2008.

TEIXEIRA, I. B. C.; SILVA, A. G. Nem só de pão vive o homem: a realidade alimentar no rural nordestino. In: CONGRESSO DA SOCIEDADE BRASILEIRA DE ECONOMIA, ADMINISTRAÇÃO E SOCIOLOGIA RURAL, 45., 2007, Londrina. Anais... Londrina: UEL, 2007.

VIANA, R. P. T.; SEGALL-CORRÊA, A. M. Insegurança alimentar das famílias residentes em municípios do interior do estado da Paraíba, Brasil. Revista de Nutrição, Campinas, v. 21, p. 111-122, jul./ago. 2008. Suplemento.

WORLD HEALTH ORGANIZATION - WHO. Global Strategy on Diet, Physical Activity and Health: Fifty-Seventh World Health Assembly. Genebra, 2004.

WILLOWS, N.; VEUGELERS, P.; RAINE, K.; KUHLE, S. Associations between household food insecurity and health outcomes in the Aboriginal population (excluding reserves). Health Reports, Ottawa, v. 22, n. 2, p. 15-20, 2011. Component of Statistics Canada Catalogue no. 82-003-X. 